\title{
The Application of Face Recognition System
}

\author{
Sai Wang ${ }^{1, *}$ \\ ${ }^{1}$ Beijing Jingshan School Caofeidian Branch, Tangshan Hebei Province, 063202 \\ *Corresponding author. Email:kiana04@qq.com\&K710903@163.com
}

\begin{abstract}
With the rapid development of computers and the internet, information security is particularly important. In the past, identification is mainly used with passwords or ID cards, but it is easy to be stolen or forgotten. However face recognition systems can quickly and simply solve the problem, so currently, it begins to be widely used in various field. This passage will mainly introduce the application of face recognition system by reading a lot of literature.

In the first section, the passage will provide the definition of face recognition system and its basic working principle. It is reported that the face recognition system has developed from simple rule-based recognition to mechanized automatic recognition. Afterwards, the application of face recognition system in various aspects will be introduced in this passage, it includes five broad applications in the financial field, education field, transportation field and public security field. In conclusion, now the world becomes more and more better because of the advance in science and technology, so face recognition is slowly recognized by people, and we also began to use it in different fields.
\end{abstract}

Keywords: Face recognition system, applications, information security, identification, mechanized automatic recognition

\section{INTRODUCTION}

Current technology development is undergoing very rapid progress, and the aim is making the life of people more and more simple, such as biometrics systems which is basically a pattern recognition system, through the use of different biometrics to identify a person, such as fingerprint, retina scanning, iris scanning and face recognition,etc. This passage attempts to describe the applications of face recognition systems in different fields, it includes financial fields, education fields, transportation fields and public security fields. And in this passage, the definition and basic principles of face recognition systems will be briefly mentioned.[4]

\section{ANALYSIS}

\subsection{The overview of face recognition system}

\subsubsection{The research and definition of face recognition}

Face recognition is the use of human facial features to complete identification. And faces are not easy to leave traces on the media, so they cannot be stolen and security is high. And compared with other biometric recognition, face recognition is more natural and intuitive, it accords with the habit of human beings to distinguish identity. And the devices of face recognition are very simple. Under the natural light, it just needs a household camera to complete it.

Face recognition refers to the input of a static image or a dynamic video. It involves segmenting an image from a static image or video sequence, and using a known database of static or dynamic faces to determine the identity of the person on the scene.

The first step is usually facing detection and segmentation, but it needs to identify whether the data contains a face. Then, the second step is face recognition, which uses segmtioned face image or image sequence to identify the testers.[2]

\subsubsection{The basic working principle of face recognition system}

A facial recognition system is a typical example of a general model, so it has a set process to do the job, as shown in Figure 1.[1] 


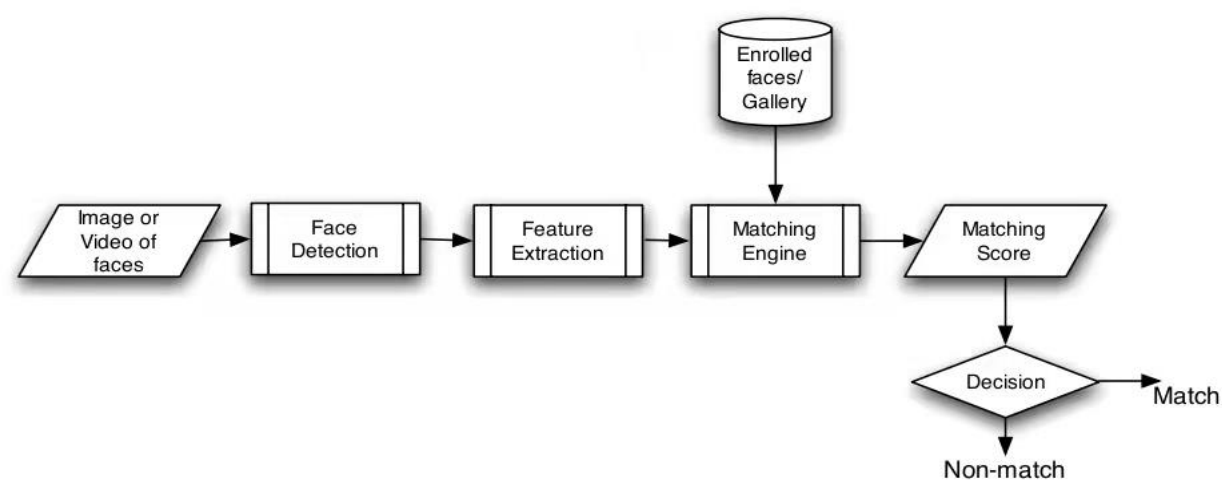

Figure 1 The basic flow of a recognition system

\subsubsection{The basic method of calculation for face recognition system}

Correlation vector machine (RVM) is a kernel function algorithm based on Bayesian theory that can handle regression and classification problems. The RVM algorithm adopts the full probability framework and introduces the prior probability into the model weight. The super parameter carries on a weight, while the value of the hyperparameter is obtained by iterating over the data. In practice, most values are lower. The empirical distribution is approximately zero, which indicates that the RVM model is sparse. According to the principle of autocorrelation, and the rest are not zero. The training vector associated with the weight is called the correlation vector.[10]

In pattern recognition, many problems are not just two types of problems, but multiple types of patterns. For example, fingerprint identification, voice recognition. In these problems, RVM classifier cannot be directly used to achieve multiple classification. To solve the problem of multi-class pattern discrimination, it is necessary to use multiple RVMS combinatorial classification method of classifier, such as "one to many" classification, "one to one" classification and so on. Then they will be talked about in the this chapter:[9]

\subsubsection{One against rest classification.}

This method is also known as "One Versus All" (OVA) classification, and the number of all categories is set as $\mathrm{K}$. The idea of OVA method is to construct $\mathrm{K}$ two-class classifiers, where the ith RVM classifier is trained on the sample set of the ith class sample is negative and the rest is positive. The number of classes in order to achieve the classification criterion, it should use multiple two class classifier combination, each of these classifiers can be one of the category with the rest of the all kinds of don't separate, this is equivalent to the one sample as a separate category, samples of all the rest of the class as a whole as the other categories, two classes of such multiple discriminant problem.Next, the category of sample $\mathrm{X}$ to be tested is determined according to multiple dichotomies.

For the multi-classification problem with $\mathrm{k}$ training samples, it is necessary to construct a classification hyperplane between class $\mathrm{i}$ and the remaining $\mathrm{k}-1$ categories. The subclassifier is K-1 second-class discriminant RVM classifier, and the optimization problem corresponding to the i-th RVM classifier can be expressed by (1).

$$
\left\{\begin{array}{c}
\min _{w_{i}, b_{i}, \xi_{i}} \frac{1}{2}\left\|w_{i}\right\|^{2}+C \sum_{j=1}^{l} \xi_{i, j} \\
\text { s.t. }\left\langle w_{i}, \boldsymbol{x}_{j}\right\rangle+b_{i} \geq 1-\xi_{i}, \quad \text { if }, \quad y_{j}=i \\
\xi_{i} \geq 0, j=1,2, \ldots, l
\end{array}\right.
$$

Obviously, The RVM classifier based on OAR classification is a multi-classification problem with $\mathrm{K}$ categories and k-1 advantages. It corresponds to K-1 classification decision function, as is shown in (2). 


$$
\left\{\begin{array}{c}
f_{1}(\boldsymbol{x})=\operatorname{sign}\left(\left\langle w_{1}, \boldsymbol{x}\right\rangle+b_{1}\right) \\
\ldots \ldots \\
f_{k}(\boldsymbol{x})=\operatorname{sign}\left(\left\langle w_{k}, \boldsymbol{x}\right\rangle+b_{k}\right)
\end{array}\right.
$$

The judgment method for category yi of an unknown sample $\mathrm{xi}$ is to substitute the feature vector $\mathrm{xi}$ corresponding to the sample into $\mathrm{k}$ classification decision functions in equation Figure 5, compare the values of each decision function, and classify the values of the classification function $\mathrm{fi}(\mathrm{x}), \mathrm{i}=1,2 \ldots$, the category with the largest $\mathrm{m}$ is judged as the category of sample $\mathrm{xi}$, as shown in (3).

$$
\operatorname{Class}(\boldsymbol{x})=\arg \max _{i=1, \ldots, k}\left(\left\langle w_{i}, \boldsymbol{x}\right\rangle+b_{i}\right)
$$

The advantages of OAR-RVM classifier are that the number of classification functions (no more than the number of categories) is small and the multi-classification speed is relatively fast. The disadvantages are that each discrimination requires to divide multiple types of samples into one category, which requires a relatively long training time, and there may be rejection areas where the test samples belong to multiple or none of the categories.[9]

\subsubsection{One against one classification}

This method is also called "voting method". Suppose that the samples to be classified are still $\mathrm{k}$ categories, and any two categories of the $\mathrm{K}$ categories can form a basic RVM dichotomies. All the training samples are classified in pairs, so that the total number of pairs of $\mathrm{K}$ categories can form $[\mathrm{K}(\mathrm{K}-1)] / 2 \mathrm{RVM}$ dichotomies. Any two kinds of samples constitute a learning machine, so the training sample set is divided into $[\mathrm{K}(\mathrm{K}-1)] / 2$ subsets, each RVM classifier only carries out machine training on its corresponding sample subset, and obtains multiple hyperplanes to form $[\mathrm{K} \quad(\mathrm{K}-1)] / 2 \quad \mathrm{RVM}$ classifiers.In the classification test, every sample to be tested is discriminated by all $[\mathrm{K}(\mathrm{K}-1)] / 2$ classifiers using the method of voting. For example, in the classification between $\mathrm{I}$ and $\mathrm{J}$, if the machine discriminates that it belongs to class I, 1 vote is added to class I; otherwise, 1 is added to the vote of class J until all the classifiers are completed. Finally, the category with the most votes is counted as the category of the test sample. This is the basic idea of "one-to-one" classification.[11]

\subsection{The application about face recognition system in five different fields}

\subsubsection{Financial field}

The application of face recognition in the financial field is mainly reflected in the following aspects:

\subsubsection{Safety prevention and control}

Now financial sector, as an important unit of security, needs a large amount of manpower and material resources in security, and the main one is realizing the real-time monitoring of multiple moving targets in dynamic scenarios. Face recognition system can solve this problem easily, and there are two advantages about it, The first one is video monitoring of business personnel: it can identify the person with face cover, and then confirm the identify of the person through the data in the public security system, so as to obtain evidence and contact the police to arrest the prisoner.And the second one is identification of personnel in the service reservoir area: the operation of the company has a certain degree of strictness, so it is also very critical to confirm the identity of the people in and out, such as the identification of the company in and out, the identification of the goods. Because if a bad person get into the company because of bad monitor system, it can have a huge impact on every aspect of the company. In these areas, face recognition can meet their needs.[3]

\subsubsection{Promoting transactional business application scenarios}

The first one is remoting to open an account:in order to better prevent risks, face-to-face account opening procedures have always been an important node in bank account opening. But this method wastes the time of customers and the process is slow. So banks can use facial recognition systems in this area, customers can not only complete the necessary procedures online, but also reduce the risk of account theft.[3]

\subsubsection{Education field}

Firstly, the attendance system is a very significant thing in education, it can serve as proof of student presence. And in some countries, the attendance of a student determines whether a student is entitled to a lecture final exam. And the application about face recognition in education is built on a desktop basis, using Python programming language, Emgu CV library 
and SQLlite relational database management system. Emgu CV Library is used as a tool to implement the feature surface method, which is used in the process of student attendance detection and face recognition. And through this method, it is not only helping teachers to monitor student, but also beneficial for students to cultivate a good habit.[4] Then if face recognition system is applied in the interact study of children, it will have advantages in the following aspects:

One is naturalness. Through the recognition of the basic biometric features of the face, and the learning content to interact with the acquisition feedback, which uses the natural interaction of the face, conforms to children's cognitive and behavioral habits, and reduces children's learning stress and expense

Second, non-contact. Face-based interactions allow users to interact without directly touching the device process, to avoid the user long time interactive operation on fingers, wrists, eyes and other joints or organ damage.

The third is fun. The camera captures and tracks face data in real time, and dynamically superimposes the effect on the user's face. Users can realize the switch of dynamic effect according to the movement of the five senses, which is easy to stimulate the interesting interactive form

User's interactive interest, prolong the interaction time. [7]

\subsubsection{Transportation field}

There are some advantages and examples about face recognition applying in transportation:

\subsubsection{Quick security check.}

Due to the large passenger flow in the passenger station, railway station and subway station, it takes a long time to manually check the passenger identity on the platform, and there are misjudgment cases due to fatigue of the staff. In order to avoid the mismatch between the person, the certificate and the ticket, face recognition technology can be applied to improve. In this case, the device can be installed in a fixed location, the ID card and the user's face can be verified at the same time, can quickly complete the comparison, saving staff and customer time.

\subsubsection{Pay by brushing your face}

For bus travel, due to the payment terminal installed on the vehicle received various aspects of interference, face recognition application environment is complex to face recognition technology maturity, fault tolerance rate requirements are higher. Face payment, face recognition payment function is opened before customers and face registration is recorded. When users take public transportation, real-time detection and comparison with the database, and payment and deduction are completed from the corresponding account. At present, some cities have started pilot face payment in public transportation. For example, Shanghai, Shenzhen, Guangzhou,etc.

\subsubsection{Traffic scheduling}

Using the security video surveillance of traffic stations and buses, combined with face recognition.[5]

\subsubsection{Security control}

In the dangerous goods transport vehicle, under certain scenarios such as long-distance bus, the driver's face recognition technology can be used to prevent fatigue driving to reduce traffic accidents. In addition, in the face of vehicle parking, traffic violations, drunk driving, super member, red light and other traffic violations, the use of face recognition technology can effectively play the role of shock.[8]

\subsubsection{The public security field}

Nowadays,high-tech applications such as big data platforms and face recognition have improved the effectiveness of case detection. But also in the maintenance of national security, public safety and the fight against terrorism played a pivotal role. Through Gabor transform, mathematical change, double attribute the public security organs on the run from the database of images and face to be recognized. Comparing the pictures, we can catch the fugitives. Then, from Table 2, the process of comparing with feature recognition face image is presented.[6] 


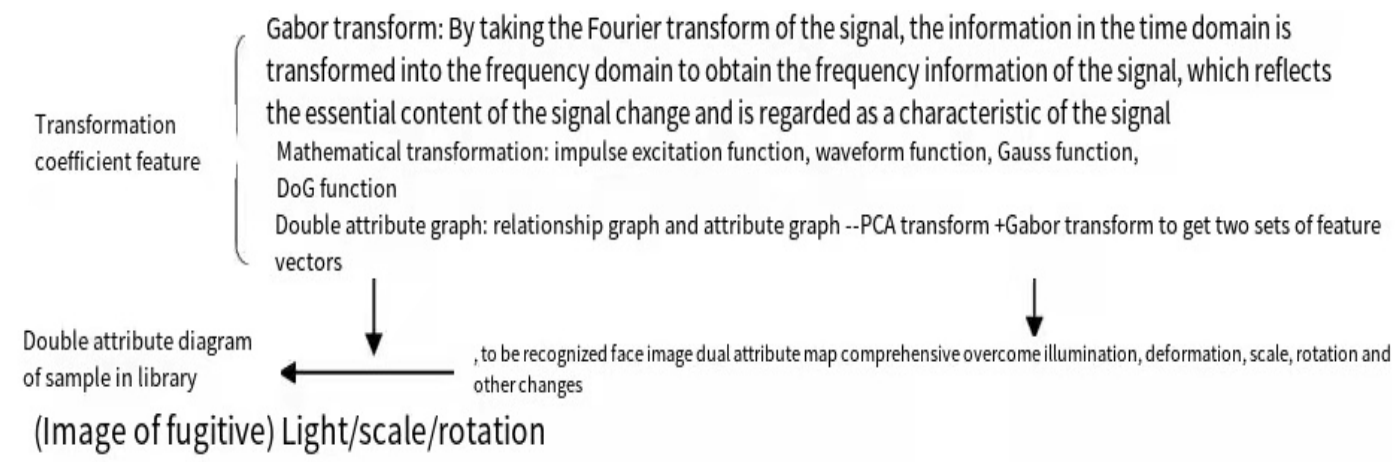

Figure 2. Comparison process between fugitive images and face images to be recognized

Although face recognition technology has been applied to real identity identification, there are still some problems.

(1) Because face recognition technology is in the development stage, on the one hand, there are problems such as algorithm improvement, automatic image registration technology, the other side is also affected by the hardware conditions, the complexity of the algorithm, algorithm recognition rate, human face light mode, expression, posture random, Therefore, the effect of practical application in the field of public security will be reduced, especially it is in the real world people are dynamic, and on the technology of dynamic face recognition research is less, there is lack.

(2) The public security organs used in the database of fugitives, criminal database stored to be arrested (database data based on the wanted or fugitives) information, but generally only a positive image, only this image is sometimes difficult to compare with the reality of the face image found.[6]

\section{CONCLUSION}

Face recognition system is now developing faster and faster, more and more used in people's lives.

This paper discusses the definition of human recognition face recognition: it is a biometric identification technology based on the facial features of people, by using a camera to capture images of a person's face and identify them through a series of processes. Then, a picture is used to describe the working progress of the face recognition system. The last and most important one is to introduce the application of face recognition in four fields:

$<1>$ Financial field. This section introduces the face recognition in company attendance, security and bank business.

$<2>$ Education field.This section focuses on an application of human recognition in recording school attendance and the interactive learning of children.
$<3>$ Transportation field. This part mainly describes the face recognition in traffic application examples and benefits.

$<4>$ The public security field. This part tells about some real examples of face recognition applied to the public security field, and its disadvantages when applied to the public security.

Therefore, through this paper, it can show that the face recognition system is more and more favored by people in various aspects, and its performance is also very consistent with people's waist, but there are still some disadvantages that need to be improved.

\section{ACKNOWLEDGMENTS}

In the process of writing a paper, I gained a lot, such as knowledge and experience. Here, I want to thank my program professors, teaching assistants, thesis teachers and English teachers for giving me the right guidance, and my family members for giving me the warm company so that I can focus on writing the paper.

\section{REFERENCES}

[1] Rawlinson, T., A. Bhalerao , and W. Li . "Principles and Methods for Face Recognition and Face Modelling." Igi Global (2010).

[2] Dongmei Wei, Spharse representation of the face, classification. Diss. Shandong University, 2016.

[3]Hong Zhang, "The application of facial recognition technology in the financial field", The Economist No. 351.05(2018):138-139

[4] Sukmandhani, A. A. , and I. Sutedja . "Face Recognition Method for Online Exams." 2019 International Conference on Information Management and Technology (ICIMTech) 2019.

[5] Zhi Deng, Et al. "Application and exploration of face recognition technology in urban traffic field." Digital Technology and Application V. 38No.355.01(2020):77-79 
[6] Jun Xiao. "Research on the application of face recognition technology in public security." Journal of Computer Science 43.022(2016):127-132

[7] Chen Zeng. Interactive learning system for children based on face recognition. Beijing University of Technology.

[8] Yujuan Chen, Shumei Yang, and Yibing Wang. "Research on the Application of Bar Code System in Enterprise ERP System." Wireless Internet Technology 000.004(2016):57-58.

[9] Changyuan Liu. Research and application of multi-classification algorithm of correlation vector machine. Diss. Harbin Engineering University, 2014.

[10] Guopeng Yang, Xin Zhou, and Xuchu Yu. "Study on Sparse Bayesian Model and Correlation Vector Machine Learning." Journal of Computer Science 07(2010): 225-228

[11] Schwenker F. Hierarchical support vector machines for multi-class pattern recognition[C]// Fourth International conference on Knowledge- Based Intelligent Engineering Systems \& Allied Technologies. Brighton, UK, 2000. 